\title{
Modelling Kidney Outcomes Based On MELD Eras - Impact of MELD Score In Renal Endpoints After Liver Transplantation
}

Paulo Ricardo Gessolo Lins ( $\nabla$ paulo.lins@unifesp.br)

Federal University of São Paulo

Roberto Camargo Narciso

Hospital Israelita Albert Einstein

Leonardo Rolim Ferraz

Hospital Israelita Albert Einstein

Virgilio Gonçalves Pereira

Hospital Israelita Albert Einstein

Ben-Hur Ferraz-Neto

Hospital Israelita Albert Einstein

Marcio Dias Almeida

Hospital Israelita Albert Einstein

Bento Fortunato Cardoso Dos Santos

Hospital Israelita Albert Einstein

Oscar Fernando Pavão

Hospital Israelita Albert Einstein

Júlio Cesar Martins Monte

Hospital Israelita Albert Einstein

Marcelino Souza Durão Júnior

Hospital Israelita Albert Einstein

Marcelo Costa Batista

Hospital Israelita Albert Einstein

\section{Research Article}

Keywords: kidney, injury, cirrhotic patients, transplants, MELD

Posted Date: September 15th, 2021

DOI: https://doi.org/10.21203/rs.3.rs-835660/v1 
License: (c) (i) This work is licensed under a Creative Commons Attribution 4.0 International License. Read Full License 


\section{Abstract}

Background: Acute kidney injury is a common complication in solid organ transplants, notably liver transplantation. The MELD is a score validated to predict mortality of cirrhotic patients, which is also used for organ allocation, however the influence of this allocation criteria on AKI incidence and mortality after liver transplantation is still uncertain.

Methods: This is a retrospective single center study of a cohort of patients submitted to liver transplant in a tertiary Brazilian hospital: Jan/2002 to Dec/2013, divided in two groups, before and after MELD implementation (pre MELD and post MELD). We evaluate the differences in AKI based on KDIGO stages and mortality rates between the two groups.

Results: 874 patients were included, 408 in pre-MELD and 466 in the post MELD era. The proportion of patients that developed AKI was lower in the post MELD era ( $p$ 0.04), although renal replacement therapy requirement was more frequent in this group $(p<0.01)$. Overall mortality rate at 28,90 and 365 days was respectively $7 \%, 11 \%$ and $15 \%$. The 1 -year mortality rate was lower in the post MELD era $(20 \%$ vs. $11 \%$, $p<0.01)$. AKI incidence was $50 \%$ lower in the post MELD era even when adjusted for clinically relevant covariates $(p<0.01)$.

Conclusion: Liver transplants performed in the post MELD era had a lower incidence of AKI, although there were more cases requiring dialysis. 1-year mortality was lower in the post MELD era, suggesting that patient care was improved during this period.

\section{Background}

Liver transplantation (LT) is preferred therapy for individuals with advanced chronic liver disease and those with acute liver failure ${ }^{1}$. Disparities among grafts distribution and patient selection force transplant polices to draw a specific liver allocation criterion that include MELD score ${ }^{2-5}$. From the past 10 years, this score has been used by the UNOS, Eurotransplant for prioritizing allocation of liver transplants and Brazilian liver allocation policy instead of the previous CPT score ${ }^{2,6,7}$.

MELD score based liver allocation policy continues under discussion, especially because some authors criticize that patients with remarkably high scores are too sicky to undergoing liver transplantation ${ }^{7}$. Other studies call attention to the increasing prevalence of hepatocellular carcinoma that could lead patients without this condition to receive a liver transplant late - because hepatocellular carcinoma diagnosis supplements MELD scores irrespective to laboratorial data ${ }^{8}$. On the other hand, this allocation policy can reduce the time on the waiting list for LT and mortality from all causes of the patient with endstage liver disease ${ }^{9,10}$.

Patients undergoing LT frequently experience some degree of AKI through the perioperative period of LT 11. This AKI could be explained by liver transplantation by itself plus additional insults to the kidney, such 
as hemorrhage $^{12}$, administration of nephrotoxic drugs ${ }^{12,13}$, post reperfusion syndrome ${ }^{14}$, hypotension ${ }^{15}$ and often substantial blood transfusion ${ }^{16}$. Although kidney injury is usually reversible, it implies some complications, such as increased length of hospitalization, longer mechanical ventilation time, sepsis, and progression to chronic renal failure, directly contributing to lower liver graft and patient survival ${ }^{16,17}$.

Serum creatinine is a cornerstone of AKI diagnosis in general population ${ }^{11}$. Despite interferences in endstage liver disease and post LT, this test is still consistent for AKI diagnosis in these setting ${ }^{18}$. Also, MELD score employs serum creatinine as a variable, and its known that end stage liver disease patient with AKI is more likely to receive a higher priority in transplantation list based on MELD score ${ }^{19}$.

Few studies report time disparities in kidney disfunction after LT based on MELD score-based liver allocation policy implementation. Lee et al. failed to demonstrate differences between three ages (19962000 / 2001-2005 / 2006-2008) in identifying chronic kidney disease in a cohort of 431 patients after 6 months of $L T^{20}$. Leithead et al. shows increase in incidence of AKI over 3 study periods (2000-2003, 2004-2007 and 2008-2011) even after adjusted for confounding variables ${ }^{21}$. However, this United Kingdom cohort adopts LT allocation policy with a different model - the UKELD score-based model for LT, a slightly different score compared with MELD score ${ }^{5}$.

Considering the high incidence of AKI in LT recipients, we proposed this observational study to evaluate the impact of MELD score-based liver allocation policy implementation on AKI diagnosis, classification, RRT requirement, and mortality.

\section{Methods}

This study was approved by local ethics committee of the Hospital Israelita Albert Einstein and informed consent were waived by the same committee - (number 00737118.6.0000.0071), São Paulo, Brazil. The study protocol agrees with the good clinical practices guideline of the Declaration of Helsinki.

\section{Data collection}

Data were collected from a series of liver transplants performed at the Hospital Israelita Albert Einstein, São Paulo, Brazil from January 2002 to December 2014 from living and deceased donors. Patients extracted until December 2006 were labelled as pre-MELD ERA, while patients after January 2007 as post MELD ERA. All transplantation recipients were admitted to the ICU in the immediate postoperative period. Before January 2007, the allocation of organs for liver transplantation was done by waiting time on the list, and subsequently started to be based on the MELD score. ${ }^{22}$. This study adheres to the Strengthening the Reporting of Observational Studies in Epidemiology guidelines for the reporting of cohort studies STROBE ${ }^{23}$

Medical records for patients undergoing LT from 2002 to 2014 were retrospectively reviewed to reclaim hospitalization clinical data, including baseline demographic characteristics and comorbidities, 
preoperative clinical and laboratory records, main indication of LT, liver disease stage according to the CPT and MELD score, and intraoperative variables including liver graft donor type, surgical time, total ischemia period, vasopressor and transfusion requirement. Admission eGFR was calculated by MDRD equation as formerly recommended for cirrhotic patients ${ }^{11}$.

A single surgical team, all particularly trained in LT, performed all procedures. The immunosuppressive routine lay on a calcineurin inhibitor (cyclosporine predominantly until 2005 and tacrolimus later), an antiproliferative drug (mycophenolate) and a corticosteroid. Whole blood levels of calcineurin inhibitor were measured by fluorescence polarization immunoassay.

Postoperative variables included the development of sepsis, need for vasopressor drugs, use of wellrecognized nephrotoxic agents, including radiocontrast agents, nonsteroidal anti-inflammatory drugs, and antimicrobials (vancomycin, aminoglycosides, polymyxin B and amphotericin B). APACHE II score or SAPS3 were determined at the time of admission to the ICU ${ }^{24,25}$. MELD score was calculated according to the equation described by Kamath and coworkers ${ }^{3,4}$. Sepsis was defined consistent with the international consensus definition ${ }^{26}$. Severe liver graft dysfunction included primary nonfunction and graft dysfunctions according to a former described criterion for liver failure 27,28 .

AKI was defined according to the AKI KDIGO recommendations ${ }^{29}$. Exact dates of AKI event and stage reached in seven days were obtained by computerized scanning of the results of daily laboratory tests for serum creatinine and 24-h urinary output from the electronic record system for each LT as the index event. RRT was initiated at the discretion of the nephrology team, based on common clinical indications such as hypervolemia, hyperkalemia, refractory acidosis, uremic sign or symptoms, and/or anuria. All attending nephrologists were part of the same group. Patients who developed AKI before LT or required RRT prior to LT were excluded from this analysis, as well as those receiving simultaneous kidney and liver transplant or who underwent liver re-transplantation. The main predictor variable of interest for the primary outcome was the period which LT were performed (Before or after MELD score-based liver allocation policy implementation, Pre-MELD and Post MELD, respectively).

The primary outcome in this analysis was AKI development after LT. Secondary outcomes were death for any cause, RRT requirement, AKI stages and RRT duration.

\section{Statistical analysis}

Numerical variables were labelled by median and interquartile range, and categorical variables by absolute and relative frequencies. For appraisal of baseline characteristics regarding the main predictor variable and primary outcome, univariable analysis was performed with the chi-squared test and MannWhitney $\mathrm{U}$ test for categorical and continuous variables, respectively. A multiple imputation approach was used to create homogeneity among APACHE 2 and SAPS 3 scores - the first were ICU elected score until December 2011, while SAPS 3 surpass this one after January 2012. 
Univariable analysis was performed to identify additional variables associated with primary outcome as potential confounders, with each variable in the database entered a logistic regression as a single covariate with AKI diagnosis as dependent variable. Those variables with a $p$ value $<0.1$ in univariable analysis or with undoubtedly clinical relevance were subsequently entered into a multivariable logistic model. A Cox proportional hazard model were performed within the main predictor (MELD era), clinical variables and AKI classification as covariates and one-year survival as independent variable. All tests were performed using SPSS version 26.0 (SPSS, Chicago, IL).

\section{Results}

Among 991 patients, 874 were included in final analysis, 408 (41.7\%) patients were labelled as pre-MELD group while $466(58.3 \%)$ as post-MELD group (Fig. 1). Baseline characteristics of patients are provided in Table 1 according to MELD era. Our results show no differences between age, gender, albumin level and admission eGFR between groups. Post MELD era shows a higher prevalence of hypertension, diabetes, hepatocellular carcinoma, and non-hepatitis virus and/or alcoholic liver disease as etiology of liver disease (Fig. 1). Also, post MELD era patients have a higher BMI and calculated MELD score. Pre-MELD era shows a higher proportion in living donor transplantation, lower total ischemia times and higher total procedure time. During in-hospital post-transplant care, there were no differences in sepsis diagnosis. Post MELD patients experimented lower first week tacrolimus concentration and lower nephrotoxic drugs exposure. A higher proportion of post MELD patients were needed vasopressors drugs, during and in the first week after liver transplantation (Table 1). 
Table 1

Basal patient characteristics according to MELD era. Data are expressed as median [IQR] or percentage. BMl: body mass index, APACHE 2: Acute Physiology and Chronic Health Evaluation II, SAPS 3: Simplified Acute Physiology Score III, eGFR: estimated Glomerular filtration rate, MELD: Model for End-Stage Liver Disease, CPT: Child-Pugh-Turcote, ICU: Intensive Care Unit

\begin{tabular}{|c|c|c|c|}
\hline & PRE-MELD ERA & POST MELD ERA & $P$ value \\
\hline & $N=408$ & $N=466$ & \\
\hline Age, years & $52.5[45.25 ; 60]$ & $54[45 ; 61]$ & 0.11 \\
\hline Gender, male (\%) & $266(65)$ & $328(70)$ & 0.18 \\
\hline $\mathrm{BMI}\left(\mathrm{Kg} / \mathrm{m}^{2}\right)$ & $25.2[22.6 ; 28]$ & $25.95[23.1 ; 29.8]$ & 0.02 \\
\hline Hypertension (\%) & $31(8)$ & $139(30)$ & $<0.01$ \\
\hline Diabetes (\%) & $79(8)$ & $139(30)$ & 0.02 \\
\hline APACHE 2 & $16[13 ; 20]$ & $17[15 ; 18]$ & 0.01 \\
\hline SAPS 3 & $39[36 ; 42]$ & $39[28 ; 53]$ & 0.83 \\
\hline Admission eGFR (mL/min/1.73m²) & $91.5[72 ; 110]$ & $88[57 ; 125]$ & 0.46 \\
\hline \multicolumn{4}{|l|}{ Cause of liver disease } \\
\hline Hepatitis C (\%) & $190(47)$ & $209(45)$ & 0.63 \\
\hline Hepatitis B (\%) & $40(10)$ & $30(6)$ & 0.08 \\
\hline Alcoholic liver cirrhosis (\%) & $83(20)$ & $92(20)$ & 0.87 \\
\hline Others (\%) & 75(18) & $122(26)$ & 0.01 \\
\hline Hepatocellular carcinoma (\%) & $94(23)$ & $180(39)$ & $<0.01$ \\
\hline Familial amyloid polyneuropathy (\%) & $17(4)$ & $22(5)$ & 0.74 \\
\hline Pre-Transplant Albumin level (g/dL) & $3.1[2.7 ; 3.4]$ & $3[2.6 ; 3.4]$ & 0.14 \\
\hline CPT Score & & & $<0.01$ \\
\hline A or non-cirrhotic (\%) & $70(17)$ & $54(12)$ & \\
\hline B (\%) & $203(50)$ & $209(45)$ & \\
\hline C (\%) & $135(33)$ & $203(44)$ & \\
\hline MELD score & $14[10 ; 18]$ & $18[11 ; 25]$ & $<0.01$ \\
\hline Preoperative Creatinine (mg/dL) & $0.9[0.7 ; 1.07]$ & $0.92[0.7 ; 1.32]$ & 0.01 \\
\hline Preoperative Total bilirubin (mg/dL) & $2.6[1.7 ; 4.6]$ & $3[1.8 ; 7 ; 5]$ & $<0.01$ \\
\hline Preoperative prothrombin time, INR & $1.6[1.4 ; 1.96]$ & $1.7[1.35 ; 2.22]$ & 0.14 \\
\hline
\end{tabular}




\begin{tabular}{|lllc|}
\hline & PRE-MELD ERA & POST MELD ERA & P value \\
\hline Surgical aspects & & & \\
\hline Deceased donor (\%) & $252(62)$ & $466(100)$ & $<0.01$ \\
\hline Piggyback technique (\%) & $389(95.3)$ & $464(99.6)$ & $<0.01$ \\
\hline Operation time (h) & $7.55[6.67 ; 8.5]$ & $6[5.17 ; 6.94]$ & $<0.01$ \\
\hline Total ischemia time (h) & $6.77(2.70 ; 10.15]$ & $9.08[7.87 ; 10.67]$ & $<0.01$ \\
\hline Vasopressor (\%) & $40(9.8)$ & $315(67.6)$ & $<0.01$ \\
\hline Number of blood packs & $4[2 ; 8]$ & $3[2 ; 7]$ & 0.18 \\
\hline Post-transplant care & & & 0.18 \\
\hline Sepsis during hospitalization (\%) & $157(39)$ & $158(34)$ & $<0.01$ \\
\hline Vasopressor after transplant (\%) & $70(17)$ & $264(30)$ & $<0.01$ \\
\hline Tacrolimus based immunosuppression (\%) & $299(73)$ & $459(98.5)$ & $<0.01$ \\
\hline First week Tacrolimus peak level (ng/mL) & $12.6[9.9 ; 15.9]$ & $5.7[3.9 ; 7.5]$ & $<0.01$ \\
\hline Acute liver rejection (\%) & $123(30)$ & $69(15)$ & $152(30)$ \\
\hline Nephrotoxic exposure (\%) & $285(70)$ & $0.55[0.35 ; 0.83]$ & $<0.01$ \\
\hline Urinary output (ml/Kg/H) & $0.25[0.15 ; 0.37]$ & 0.01 \\
\hline
\end{tabular}

As primary outcome analysis, 706 patients (81\%) fulfill KDIGO AKI criteria after liver transplant procedure, 297 (34\%) KDIGO stage 1, 186 (21.3\%) 2, and 223 (25.5\%) 3. Among study periods, AKI were present in $342(84 \%)$ and 364 (78\%) in pre and post-MELD periods, respectively ( $\mathrm{p} 0.04)$. Pre-MELD patients KDIGO AKI classification proportion were $171(41.9 \%)$ stage 1, $113(27.7 \%) 2$ and $58(14.2 \%) 3$, while post-MELD patients were $126(27 \%)$ stage 1, 73 (15.7\%) 2 and 165 (35.4\%) 3, with a higher proportion of KDIGO 2 and 3 (severe AKI) in post-MELD group $(p<0.01$, Table 3$)$. 
Table 3

Secondary outcomes according to AKI diagnosis. Data are expressed as median [IQR] or percentage. ICU: Intensive care unit, AKI: Acute Kidney Injury, RRT: Renal replacement therapy.

\begin{tabular}{|llll|}
\hline & PRE-MELD ERA & POST MELD ERA & P value \\
\hline & $\mathrm{N}=408$ & $\mathrm{~N}=466$ & \\
\hline Hospital length (days) & $15[11 ; 23]$ & $13[8 ; 22]$ & $<0.01$ \\
\hline ICU length (days) & $3[2 ; 8]$ & $2[1 ; 4]$ & $<0.01$ \\
\hline Retransplant during index hospitalization (\%) & $36(8.8)$ & $36(7.7)$ & 0.62 \\
\hline Acute liver rejection (\%) & $123(30)$ & $69(15)$ & $<0.01$ \\
\hline Acute Kidney Injury (\%) & $342(84)$ & $364(78)$ & 0.04 \\
\hline KDIGO 1 & $171(41.9)$ & $126(27)$ & $<0.01$ \\
\hline KDIGO 2 & $113(27.7)$ & $73(15.7)$ & $<0.01$ \\
\hline KDIGO 3 & $58(14.2)$ & $165(35.4)$ & $<0.01$ \\
\hline Duration of AKI (days) & $16[10 ; 23]$ & $6[2 ; 23]$ & $<0.01$ \\
\hline Peak creatinine during AKI episode (mg/dL) & $1.6[1.1 ; 2.1]$ & $1.9[1.32 ; 2.69]$ & $<0.01$ \\
\hline Dialysis (\%) & $68(17)$ & $131(28)$ & $<0.01$ \\
\hline Continuous therapies (\%) & $39(10)$ & $68(15)$ & 0.03 \\
\hline Intermittent therapies (\%) & $50(12)$ & $115(28)$ & $<0.01$ \\
\hline Time of RRT dependence (days) & $11.5[3 ; 26.75]$ & $17.5[6 ; 43.75]$ & $<0.01$ \\
\hline Hospital mortality (\%) & $33(8)$ & $36(8)$ & 0.90 \\
\hline 28 days mortality (\%) & $25(6)$ & $45(10)$ & 0.36 \\
\hline 90 days mortality (\%) & $51(13)$ & $45(10)$ & 0.19 \\
\hline 1-year mortality (\%) & $81(20)$ & $50(11)$ & $<0.01$ \\
\hline
\end{tabular}

AKI diagnosis following liver transplantation remained more frequent in patients with lower pre-transplant albumin levels, lower estimated glomerular filtration rate and higher MELD values. Otherwise, patients with hepatocellular carcinoma diagnosis shows lower AKI proportion. Higher procedure time, wider total ischemic times, sepsis diagnosis after transplantation, vasopressor exposure and nephrotoxic drug prescription were statistically more likely in patients with AKI (Table 2). 
Table 2

Basal patient characteristics according to AKI occurrence. Data are expressed as median [IQR] or percentage. BMI: body mass index, APACHE 2: Acute Physiology and Chronic Health

Evaluation II, SAPS 3: Simplified Acute Physiology Score III, eGFR: estimated glomerular filtration rate

\begin{tabular}{|c|c|c|c|}
\hline & NON-AKI & AKI & $P$ value \\
\hline & $N=168$ & $N=706$ & \\
\hline Age, years & $55[44 ; 63]$ & $53[45 ; 60]$ & 0.06 \\
\hline Gender, male (\%) & $114(67.9)$ & $480(68)$ & 0.97 \\
\hline $\mathrm{BMI}\left(\mathrm{Kg} / \mathrm{m}^{2}\right)$ & $25.7[22.6 ; 28.5]$ & $25.6[22.9 ; 29.1]$ & 0.38 \\
\hline Hypertension (\%) & $37(22)$ & $133(18.8)$ & 0.35 \\
\hline Diabetes (\%) & $41(24.4)$ & $25.6[22.9 ; 29.1]$ & 0.66 \\
\hline APACHE 2 & $17[14 ; 19]$ & $16[14 ; 19]$ & 0.90 \\
\hline SAPS 3 & $38[30.25 ; 43]$ & $39[32 ; 44,25]$ & $<0.01$ \\
\hline Admission eGFR (mL/min/1.73m²) & $95.5[76.2 ; 118]$ & $90[63 ; 121]$ & 0.01 \\
\hline \multicolumn{4}{|l|}{ Pathogenesis of liver disease } \\
\hline Hepatitis C (\%) & $75(44.6)$ & $324(45.9)$ & 0.77 \\
\hline Hepatitis B (\%) & $17(10.1)$ & $53(7.5)$ & 0.26 \\
\hline Alcoholic liver cirrhosis (\%) & $27(16.1)$ & $148(21)$ & 0.15 \\
\hline Others (\%) & $35(20.8)$ & $162(22.9)$ & 0.56 \\
\hline Hepatocellular carcinoma (\%) & $75(44.6)$ & $199(28.2)$ & $<0.01$ \\
\hline Familial amyloid polyneuropathy (\%) & $11(6.5)$ & $28(4)$ & 0.15 \\
\hline Pre-Transplant Albumin level (g/dL) & $3.1[2.9 ; 3.6]$ & $3[2.7 ; 3.3]$ & $<0.01$ \\
\hline CPT Score & & & $<0.01$ \\
\hline A or non-cirrhotic (\%) & $35(20.8)$ & $89(12.6)$ & \\
\hline B (\%) & $83(49.4)$ & $329(46.6)$ & \\
\hline C (\%) & $50(29.8)$ & $288(40.8)$ & \\
\hline MELD score & $13[8 ; 18]$ & $16[11 ; 23]$ & $<0.01$ \\
\hline Preoperative Creatinine (mg/dL) & $0.8[0.7 ; 1.0]$ & $0.9[0.7 ; 1.2]$ & 0.01 \\
\hline Preoperative Total bilirubin (mg/dL) & $2.15[1.4 ; 4.45]$ & $3[1.8 ; 5.9]$ & $<0.01$ \\
\hline Preoperative prothrombin time, INR & $1.5[1.23 ; 1.9]$ & $1.7[1.4 ; 2.15]$ & $<0.01$ \\
\hline
\end{tabular}




\begin{tabular}{|llll|}
\hline & NON-AKI & AKI & P value \\
\hline Surgical aspects & & & \\
\hline Deceased donor (\%) & $150(89.3)$ & $568(80.5)$ & $<0.01$ \\
\hline Piggyback technique (\%) & $161(95.8)$ & $692(98)$ & 0.09 \\
\hline Operation time (h) & $6[5.1 ; 7.31]$ & $7[5.67 ; 8]$ & $<0.01$ \\
\hline Total ischemia time (h) & $8.6[6.8 ; 10.6]$ & $8.3[6.4 ; 10.4]$ & 0.19 \\
\hline Vasopressor (\%) & $64(38.1)$ & $291(41.2)$ & 0.49 \\
\hline Number of blood packs & $3[1 ; 9]$ & $4[2 ; 8]$ & 0.31 \\
\hline Post-transplant care & & & $<0.01$ \\
\hline Sepsis during hospitalization (\%) & $24(14.3)$ & $291(41.2)$ & $<0.01$ \\
\hline Vasopressor after transplant (\%) & $41(24.4)$ & $293(41.5)$ & 0.08 \\
\hline Tacrolimus based immunosuppression (\%) & $153(91.1)$ & $605(85.7)$ & 0.77 \\
\hline First week Tacrolimus peak level (ng/mL) & $7.7[5.7 ; 11.8]$ & $8.1[4.9 ; 12.5]$ & $<0.01$ \\
\hline Acute liver rejection (\%) & $18(10.7)$ & $174(24.6)$ & $<0.01$ \\
\hline Nephrotoxic exposure (\%) & $56(33.3)$ & $381(54)$ & $<0.01$ \\
\hline Urinary output (ml/Kg/H) & $0.68[0.43 ; 0.97]$ & $0.36[0.21 ; 0.56]$ & $<$ \\
\hline
\end{tabular}

Renal replacement therapy was more frequent in post-MELD group, and even continuous as intermittent therapies were more prevalent in this group ( 0.03 and $<0.01$, respectively) (Table 3 ). Despite that, mortality rates during hospital stay, 28 and 90 days were similar among two groups. Surprisingly, oneyear mortality rates were lower in post-MELD group, even with higher MELD, SAPS3, KDIGO 2 and 3 AKI diagnosis as RRT needs in that group $(\mathrm{p}<0.01)$. AKI diagnosis did not impact in mortality rates (inhospital, 28, 90 and 365 days), but the length of stay in the ICU and hospital has increased. (Table 4). 
Table 4

Primary and secondary outcomes according to MELD era. Data are expressed as median [IQR] or percentage. ICU: Intensive care unit KDIGO: Kidney disease Improving Global Outcomes AKI: Acute Kidney Injury RRT: Renal Replacement Therapy

\begin{tabular}{|llll|}
\hline & NON-AKI & AKI & P value \\
\hline & $\mathrm{N}=168$ & $\mathrm{~N}=706$ & \\
\hline Hospital length (days) & $9[7 ; 12]$ & $16[11 ; 25]$ & $<0.01$ \\
\hline ICU length (days) & $2[1 ; 3]$ & $3[2 ; 6]$ & $<0.01$ \\
\hline Retransplant during index hospitalization (\%) & $10(6)$ & $62(8.8)$ & 0.23 \\
\hline Acute liver rejection (\%) & $18(10.7)$ & $174(24.6)$ & $<0.01$ \\
\hline Hospital mortality (\%) & $13(7.7)$ & $56(7.9)$ & 0.93 \\
\hline 28 days mortality (\%) & $14(8.3)$ & $48(6.8)$ & 0.50 \\
\hline 90 days mortality (\%) & $15(8.9)$ & $81(11.5)$ & 0.41 \\
\hline 1-year mortality (\%) & $17(10.1)$ & $114(16.1)$ & 0.05 \\
\hline
\end{tabular}

Table 5 shows logistic regression panels for AKI, RRT and mortality. We constructed 5 models using covariates that shows clinical relevance and/or statistical significance at univariate analysis. Our models support that post-MELD era reduces AKI diagnosis, mainly sinking AKI KDIGO 1 and 2 insults. Otherwise, these period augment RRT needs, even corrected by many covariates but shows a signal for mortality decline after logistic regression modelling - model 4 and 5 for 90 days mortality. For illustration porpoise, Fig. 2 shows each KDIGO stratum AKI risk according to MELD era group, showing that post MELD era shrinks AKI risk predominantly by KDIGO 1 and 2 reduction effect. 
Table 5

Logistic regression showing unadjusted and 5 distinctive adjusting models for AKI, RRT and 28 days mortality risk between MELD eras. BMI: Body mass index MELD: Model for end-stage liver disease, eGFR: estimated glomerular filtration rate, SAPS 3: Simplified Acute Physiology Score III.

$\begin{array}{llll}\text { Predictor } & H R & \begin{array}{ll}95 \% & P \text { - } \\ \text { value }\end{array}\end{array}$

AKI risk by performing transplantation in Post MELD era

Unadjusted

$0.68 \quad 0.48, \quad 0.03$

0.97

Model 1 - Adjusted for age and gender

$0.69 \quad 0.49, \quad 0.03$

0.97

Model 2 - Adjusted for age, gender, diabetes, hypertension, BMI and MELD

$0.590 .41<<$

$0.86 \quad 0.01$

Model 3 - Adjusted for age, gender, diabetes, hypertension, BMI, MELD and eGFR

$0.60 \quad 0.41,<$

$0.87 \quad 0.01$

Model 4 - Adjusted for age, gender, diabetes, hypertension, BMI, MELD, eGFR, Vasopressor (During and after transplant), Transfusion, First week

$0.46 \quad 0.24, \quad 0.01$

Tacrolimus peak level and Nephrotoxic drug exposure

Model 5 - Adjusted for age, gender, diabetes, hypertension, BMI, MELD, eGFR, Vasopressor (During and after transplant), Transfusion, First week

$0.49 \quad 0.26, \quad 0.02$

Tacrolimus peak level, Nephrotoxic drug exposure, Acute liver rejection,

0.92

Sepsis and SAPS 3

RRT risk by performing transplantation in Post MELD era

Unadjusted

$1.951 .40,<$

$2.71 \quad 0.01$

Model 1 - Adjusted for age and gender

$1.991 .43,<$

$2.78 \quad 0.01$

Model 2 - Adjusted for age, gender, diabetes, hypertension, BMI and MELD

$1.61 \quad 1.12,<$

$2.32 \quad 0.01$

Model 3 - Adjusted for age, gender, diabetes, hypertension, BMI, MELD and eGFR

$1.73 \quad 1.20,<$

$2.50 \quad 0.01$

Model 4 - Adjusted for age, gender, diabetes, hypertension, BMI, MELD, eGFR, $\quad 1.89 \quad 0.94, \quad 0.07$ Vasopressor (During and after transplant), Transfusion, First week 3.80

Tacrolimus peak level and Nephrotoxic drug exposure

Model 5 - Adjusted for age, gender, diabetes, hypertension, BMI, MELD, eGFR, Vasopressor (During and after transplant), Transfusion, First week

Tacrolimus peak level, Nephrotoxic drug exposure, Acute liver rejection,

Sepsis and SAPS 3

30 days mortality risk by performing transplantation in Post MELD era

Unadjusted 


\begin{tabular}{|c|c|c|c|}
\hline Predictor & $H R$ & $\begin{array}{l}95 \% \\
\mathrm{Cl}\end{array}$ & $\begin{array}{l}P \text { - } \\
\text { value }\end{array}$ \\
\hline Model 1 - Adjusted for age and gender & 1.35 & $\begin{array}{l}0.79 \\
2.29\end{array}$ & 0.26 \\
\hline Model 2 - Adjusted for age, gender, diabetes, hypertension, BMI and MELD & 1.37 & $\begin{array}{l}0.78 \\
2.40\end{array}$ & 0.26 \\
\hline $\begin{array}{l}\text { Model } 3 \text { - Adjusted for age, gender, diabetes, hypertension, BMI, MELD and } \\
\text { eGFR }\end{array}$ & 1.31 & $\begin{array}{l}0.79 \\
2.44\end{array}$ & 0.25 \\
\hline $\begin{array}{l}\text { Model } 4 \text { - Adjusted for age, gender, diabetes, hypertension, BMI, MELD, eGFR, } \\
\text { Vasopressor (During and after transplant), Transfusion, First week } \\
\text { Tacrolimus peak level and Nephrotoxic drug exposure }\end{array}$ & 0.11 & $\begin{array}{l}0.02 \\
0.52\end{array}$ & $<.01$ \\
\hline $\begin{array}{l}\text { Model } 5 \text { - Adjusted for age, gender, diabetes, hypertension, BMI, MELD, eGFR, } \\
\text { Vasopressor (During and after transplant), Transfusion, First week } \\
\text { Tacrolimus peak level, Nephrotoxic drug exposure, Acute liver rejection, } \\
\text { Sepsis and SAPS } 3\end{array}$ & 0.13 & $\begin{array}{l}0.02, \\
0.60\end{array}$ & $<.01$ \\
\hline
\end{tabular}

Figure 3 shows a cox proportional hazard analysis for 365 days mortality risk analysis. Liver transplantation after MELD policies implementation shows as protection factor, furthermore AKI KDIGO 3 classification impact negatively on patient survival, as MELD score and hepatocellular carcinoma diagnosis.

\section{Discussion}

As far as we know, this is the first time series that exclusively compares before and after MELD liver transplantation policy implementation influence on kidney outcomes. Our data shows that post-MELD period impact in fewer AKI diagnosis, however there were more severe AKI and these patients require more dialysis. Despite that, post-MELD era shows a decline in mortality rates after 1-year period.

Our data show a noticeable high incidence in AKI after liver transplantation which corroborate with previous studies that adopt KDIGO diagnostic criterion as surrogate of $A K{ }^{16}{ }^{16}$. We hypothesized that higher KDIGO sensitivity for AKI diagnosis ${ }^{30}$, more burden of comorbidities like hypertension and diabetes, and sicker patients, denoted by higher MELD scores ${ }^{13,21}$, could explain these numbers.

Surprisingly, post MELD period shows a lesser proportion in AKI diagnosis even after adjustments with 5 proposed models of logistic regression. This result could be explained by a complex interaction between dampening previous known AKI risk factors and by adopting new strategies that could mitigate kidney damage. A well-known AKI associated risk factor is surgical time, it's recognized that reducing these periods of insults could mitigate kidney insult, both in general as liver transplant surgery ${ }^{16}$. Reduced nephrotoxic agent prescription could also support our results, summarized by protocolized sepsis treatment sparing potential nephrotoxic agents or postpone/switch radiocontrast exams by another kind of radiologic propaedeutic ${ }^{31}$. Adopting tacrolimus as standard post-transplant immunosuppression agent is also a previous known strategy to amend kidney injury. In addition, better tacrolimus monitoring 
policies could also attenuate kidney injury occurrence ${ }^{32}$. However, in our analysis, the first week peak tacrolimus trough concentration was not found to be significantly associated with development of AKI, possibly because recipients who are suspected to occurrence AKI receive a low dose and/or late introduction of calcineurin inhibitor. None of these covariates individually shows impact in AKI diagnosis, but we could hypothesize that all of those together could impact in our primary endpoint.

Higher MELD and SAPS3 could impact in more severe AKI diagnosis, as previous described ${ }^{33}$, also higher liver ischemic times ${ }^{14,32,34}$ and more vasopressor requirement ${ }^{35,36}$ preclude AKI progression after liver transplantation. Briefly, sicker patients with higher vasoactive requirement and worse liver draft quality is going to experience more period and higher grade of post reperfusion syndrome ${ }^{37}$, denoting higher risk for AKI development and progression ${ }^{14,38}$.

As previously shown, when AKI develop in post MELD patients, these diagnosis incline to worse classification categories and carries more risk for renal replacement therapy requirement, even with lower AKI diagnosis proportion in this group and lower mortality. This singularities, higher dialysis requirement with lower associated mortality, had also seen in another scenarios ${ }^{39-41}$ and could be explained by protocolized watchfulness for kidney injury patterns reducing transient AKI episodes resulting in proportional higher persistent and severe AKI episodes ${ }^{39}$. Also, changing dialysis treatment patterns over the time period of the study may account for reduced mortality ratios. That is, despite lack of randomized controlled data to funding this argument, perhaps earlier and higher dose and more frequent dialysis may be beneficial ${ }^{42,43}$. Previous data from our group also shows that lower AKI exposure time could reduce mortality in post liver transplant patients ${ }^{44}$.

Liver transplant mortality rate is decreasing, which is encouraging, but it is important to recognize that all transformations had been made in care over the last decade resulted in this improvement $8,37,45,46$. It is remarkable to note that mortality in other conditions, like acute kidney injury dialysis patients has also declined over the last decade despite the paucity of randomized trials which have shown a benefit of any specific intervention ${ }^{39,42,43}$; so, it is possible that multiple changes/bundles/improvements over period have combined to improve outcomes ${ }^{47,48}$.

This study has several limitations. The retrospective design of this study has its inherent shortcomings. First, we could not determine AKI etiology in part of absence of routine urinalysis after liver transplantation. Second, liver donor characteristics to calculate donor risk index, post-reperfusion syndrome temporal data and associated variables were unavailable. Third, because retrospective design itself, we couldn't assert that liver allocation policies change was exclusively responsible for our results in $\mathrm{AKI}$ and one-year mortality rates reduction (about $50 \%$ in logistic and cox proportional hazards regression models, respectively). These results help to strengthen that liver transplant MELD allocation policies positively impact in non-direct correlated endpoints, like acute kidney injury.

In conclusion, MELD adoption as standard liver allocation policies reduce AKI diagnosis after liver transplantation, resulting in lower mortality rates even with higher renal replacement therapy requirement 
in these population.

\section{Abbreviations}

\begin{tabular}{ll} 
AKI & Acute Kidney Injury \\
\hline APACHE 2 & Acute Physiology and Chronic Health Evaluation II \\
\hline BMI & Body mass index \\
\hline CMV & Cytomegalovirus \\
\hline CPT & Child-Pugh-Turcote score \\
\hline CRRT & Continous Renal Replacement Therapy \\
\hline eGFR & Estimated glomerular filtration rate \\
\hline HDi & Intermittent hemodialysis \\
\hline ICU & Intensive Care Unit \\
\hline IQR & Interquartile Range \\
\hline KDIGO & Kidney Disease - Improving Global Outcomes - Acute Kidney Injury Guidelines \\
\hline LT & Liver Transplantation \\
\hline MDRD & Modification of Diet in Renal Disease \\
\hline MELD & Model for End Stage Liver Disease \\
\hline RRT & Renal Replacement Therapy \\
\hline SAPS 3 & Simplified Acute Physiology Score III \\
\hline TAC & Tacrolimus \\
\hline UKELD & United Kingdom - End Stage Liver Disease Score \\
\hline UNOS & United Network for Organ Sharing
\end{tabular}

\section{Declarations}

1. ETHICS APPROVAL AND CONSENT TO PARTICIPATE: This study was approved by the Ethics in Research Committee of the Hospital Israelita Albert Einstein and informed consent were waived by the same committee - (number 00737118.6.0000.0071).

2. CONSENT TO PUBLISH: Not applicable.

3. AVAILABILITY OF DATA AND MATERIALS: The datasets used and/or analyzed during the current study are available from the corresponding author upon reasonable request. 


\section{Numbers}

Total number of words (main text): 2518

Total number of figures: 3

Total number of tables: 5

\section{AUTHOR`S CONTRIBUTIONS:}

Paulo Ricardo Gessolo Lins: participated in study design, data acquisition, data analysis and interpretation, written manuscript drafting, critical revision of manuscript drafts, and final approval of manuscript for publication. Accountability for all aspects of the work, including resolution of all questions related to the accuracy or integrity of all aspects of the work.

Roberto Camargo Narciso: participated in data acquisition, data analysis and interpretation, critical revision of manuscript drafts, and final approval of manuscript for publication. Accountability for all aspects of the work, including resolution of all questions related to the accuracy or integrity of all aspects of the work.

Leonardo Rolim Ferraz: participated in study design, data acquisition, data analysis and interpretation, critical revision of manuscript drafts, and final approval of manuscript for publication. Accountability for all aspects of the work, including resolution of all questions related to the accuracy or integrity of all aspects of the work.

Virgilio Gonçalves Pereira: participated in critical revision of manuscript drafts, and final approval of manuscript for publication. Accountability for all aspects of the work, including resolution of all questions related to the accuracy or integrity of all aspects of the work.

Ben-Hur Ferraz-Neto: participated in critical revision of manuscript drafts, and final approval of manuscript for publication. Accountability for all aspects of the work, including resolution of all questions related to the accuracy or integrity of all aspects of the work.

Marcio Dias De Almeida: participated in critical revision of manuscript drafts, and final approval of manuscript for publication. Accountability for all aspects of the work, including resolution of all questions related to the accuracy or integrity of all aspects of the work.

Bento Fortunato Cardoso Dos Santos: participated in critical revision of manuscript drafts, and final approval of manuscript for publication. Accountability for all aspects of the work, including resolution of all questions related to the accuracy or integrity of all aspects of the work.

Oscar Fernando Pavão Dos Santos: participated in critical revision of manuscript drafts, and final approval of manuscript for publication. Accountability for all aspects of the work, including resolution of all questions related to the accuracy or integrity of all aspects of the work. 
Júlio Cesar Martins Monte: participated in critical revision of manuscript drafts, and final approval of manuscript for publication. Accountability for all aspects of the work, including resolution of all questions related to the accuracy or integrity of all aspects of the work.

Marcelino Souza Durão: participated in study design, critical revision of manuscript drafts, and final approval of manuscript for publication. Accountability for all aspects of the work, including resolution of all questions related to the accuracy or integrity of all aspects of the work.

Marcelo Costa Batista: participated in study design, data acquisition, data analysis and interpretation, written manuscript drafting, critical revision of manuscript drafts, and final approval of manuscript for publication. Accountability for all aspects of the work, including resolution of all questions related to the accuracy or integrity of all aspects of the work.

6. COMPETING INTERESTS: The authors declare that they have no competing interests.

7. Funding: This is retrospective analysis from an individual investigator initiative. Paulo Ricardo Gessolo Lins received a PhD's scholarship from "Coordenação de Aperfeiçoamento de Pessoal de Nível Superior" (CAPES, Brazil) for the preparation of this manuscript.

\section{Acknowledgments}

This study was supported by a grant from CAPES (Coordenação de Aperfeiçoamento de Pessoal de Nível Superior).

\section{References}

1. Mahmud N. Selection for Liver Transplantation: Indications and Evaluation. Current Hepatology Reports. 2020;19(3):203-212.

2. Schilsky ML, Moini M. Advances in liver transplantation allocation systems. World journal of gastroenterology. 2016;22(10):2922-2930.

3. Malinchoc M, Kamath PS, Gordon FD, Peine CJ, Rank J, ter Borg PCJ. A model to predict poor survival in patients undergoing transjugular intrahepatic portosystemic shunts. Hepatology (Baltimore, Md). 2000;31(4):864-871.

4. Kamath PS, Wiesner RH, Malinchoc M, et al. A model to predict survival in patients with end-stage liver disease. Hepatology (Baltimore, Md). 2001;33(2):464-470.

5. Asrani SK, Kamath PS. Model for end-stage liver disease score and MELD exceptions: 15 years later. Hepatology international. 2015;9(3):346-354.

6. Teixeira AC, Souza FF, Mota GdA, Martinelli AdLC, Sankarankutty AK, Castro e Silva Od. Liver transplantation: expectation with MELD score for liver allocation in Brazil. Acta Cirúrgica Brasileira. 2006;21:12-14.

7. Journal of Hepatology. 2019;71(4):707-718. 
8. Santopaolo F, Lenci I, Milana M, Manzia TM, Baiocchi L. Liver transplantation for hepatocellular carcinoma: Where do we stand? World journal of gastroenterology. 2019;25(21):2591-2602.

9. Mazzaferro V, Regalia E, Doci R, et al. Liver transplantation for the treatment of small hepatocellular carcinomas in patients with cirrhosis. N Engl J Med. 1996;334(11):693-699.

10. Mazzaferro V, Llovet JM, Miceli R, et al. Predicting survival after liver transplantation in patients with hepatocellular carcinoma beyond the Milan criteria: a retrospective, exploratory analysis. Lancet Oncol. 2009;10(1):35-43.

11. Durand F, Francoz C, Asrani SK, et al. Acute Kidney Injury After Liver Transplantation. Transplantation. 2018;102(10):1636-1649.

12. Park MH, Shim HS, Kim WH, et al. Clinical Risk Scoring Models for Prediction of Acute Kidney Injury after Living Donor Liver Transplantation: A Retrospective Observational Study. PLoS One. 2015;10(8):e0136230.

13. O'Riordan A, Wong V, McQuillan R, McCormick PA, Hegarty JE, Watson AJ. Acute Renal Disease, as Defined by the RIFLE Criteria, Post-Liver Transplantation. American Journal of Transplantation. 2007;7(1):168-176.

14. Kalisvaart M, Schlegel A, Umbro I, et al. The AKI Prediction Score: a new prediction model for acute kidney injury after liver transplantation. HPB. 2019;21(12):1707-1717.

15. Rueggeberg A, Boehm S, Napieralski F, et al. Development of a risk stratification model for predicting acute renal failure in orthotopic liver transplantation recipients. Anaesthesia. 2008;63(11):11741180.

16. Thongprayoon C, Kaewput W, Thamcharoen N, et al. Incidence and Impact of Acute Kidney Injury after Liver Transplantation: A Meta-Analysis. Journal of clinical medicine. 2019;8(3):372.

17. Narciso R, Ferraz L, J O Rodrigues C, et al. Low estimated glomerular filtration rate and chronic kidney failure following liver transplant: A retrospective cohort study. Vol 362013.

18. Rognant N, Lemoine S. Evaluation of renal function in patients with cirrhosis: Where are we now? World Journal of Gastroenterology: WJG. 2014;20(10):2533-2541.

19. Saxena V, Lai JC. Kidney Failure and Liver Allocation: Current Practices and Potential Improvements. Advances in chronic kidney disease. 2015;22(5):391-398.

20. Lee S, Park S, Kang MW, et al. Long-term impact of dialysis-requiring AKI during the perioperative period of liver transplantation on postdischarge outcomes. Clinical transplantation. 2019;33(8):e13649.

21. Leithead JA, Rajoriya N, Gunson BK, Muiesan P, Ferguson JW. The evolving use of higher risk grafts is associated with an increased incidence of acute kidney injury after liver transplantation. Journal of Hepatology. 2014;60(6):1180-1186.

22. Salvalaggio P, Afonso RC, Pereira LA, Ferraz-Neto BH. The MELD system and liver transplant waitinglist mortality in developing countries: lessons learned from Sao Paulo, Brazil. Einstein (Sao Paulo, Brazil). 2012;10(3):278-285. 
23. Elm Ev, Altman DG, Egger M, Pocock SJ, Gøtzsche PC, Vandenbroucke JP. Strengthening the reporting of observational studies in epidemiology (STROBE) statement: guidelines for reporting observational studies. BMJ. 2007;335(7624):806-808.

24. Knaus WA, Draper EA, Wagner DP, Zimmerman JE. APACHE II: a severity of disease classification system. Critical care medicine. 1985;13(10):818-829.

25. Moreno RP, Metnitz PG, Almeida E, et al. SAPS 3-From evaluation of the patient to evaluation of the intensive care unit. Part 2: Development of a prognostic model for hospital mortality at ICU admission. Intensive Care Med. 2005;31(10):1345-1355.

26. Singer M, Deutschman CS, Seymour CW, et al. The Third International Consensus Definitions for Sepsis and Septic Shock (Sepsis-3). JAMA. 2016;315(8):801-810.

27. Banff schema for grading liver allograft rejection: an international consensus document. Hepatology (Baltimore, Md). 1997;25(3):658-663.

28. Demetris AJ, Bellamy C, Hübscher SG, et al. 2016 Comprehensive Update of the Banff Working Group on Liver Allograft Pathology: Introduction of Antibody-Mediated Rejection. American Journal of Transplantation. 2016;16(10):2816-2835.

29. Kidney Disease: Improving Global Outcomes (KDIGO) Acute Kidney Injury Work Group KDIGO Clinical Practice Guideline for Acute Kidney Injury. Kidney Int Suppl. 2012;1(2):1-138.

30. Pan H-C, Chien Y-S, Jenq C-C, et al. Acute Kidney Injury Classification for Critically III Cirrhotic Patients: A Comparison of the KDIGO, AKIN, and RIFLE Classifications. Scientific reports. 2016;6:23022-23022.

31. Ozkok S, Ozkok A. Contrast-induced acute kidney injury: A review of practical points. World journal of nephrology. 2017;6(3):86-99.

32. Sharma P, Sun Y, Neal J, et al. Renal Outcomes of Liver Transplantation Recipients Receiving Standard Immunosuppression and Early Renal Sparing Immunosuppression: A Retrospective Single Center Study. Transplant Direct. 2019;5(9):e480.

33. Nadeem A, Salahuddin N, El Hazmi A, et al. Chloride-liberal fluids are associated with acute kidney injury after liver transplantation. Critical care (London, England). 2014;18(6):625.

34. Sharma P, Sun Y, Neal J, et al. Renal Outcomes of Liver Transplantation Recipients Receiving Standard Immunosuppression and Early Renal Sparing Immunosuppression: A Retrospective Single Center Study. Transplant Direct. 2019;5(9):e480-e480.

35. Wyssusek KH, Keys AL, Yung J, Moloney ET, Sivalingam P, Paul SK. Evaluation of perioperative predictors of acute kidney injury post orthotopic liver transplantation. Anaesth Intensive Care. 2015;43(6):757-763.

36. Karapanagiotou A, Dimitriadis C, Papadopoulos S, et al. Comparison of RIFLE and AKIN criteria in the evaluation of the frequency of acute kidney injury in post-liver transplantation patients. Transplantation proceedings. 2014;46(9):3222-3227.

37. Kassel CA, Fremming BA, Brown BA, Markin NW. 2019 Clinical Update in Liver Transplantation. Journal of cardiothoracic and vascular anesthesia. 2020. 
38. Umbro I, Tinti F, Scalera I, et al. Acute kidney injury and post-reperfusion syndrome in liver transplantation. World journal of gastroenterology. 2016;22(42):9314-9323.

39. Brown JR, Rezaee ME, Hisey WM, Cox KC, Matheny ME, Sarnak MJ. Reduced Mortality Associated with Acute Kidney Injury Requiring Dialysis in the United States. American Journal of Nephrology. 2016;43(4):261-270.

40. Grams ME, Waikar SS, MacMahon B, Whelton S, Ballew SH, Coresh J. Performance and limitations of administrative data in the identification of AKI. Clin J Am Soc Nephrol. 2014;9(4):682-689.

41. Hsu RK, McCulloch CE, Dudley RA, Lo LJ, Hsu CY. Temporal changes in incidence of dialysisrequiring AKI. J Am Soc Nephrol. 2013;24(1):37-42.

42. Zarbock A, Kellum JA, Schmidt C, et al. Effect of Early vs Delayed Initiation of Renal Replacement Therapy on Mortality in Critically III Patients With Acute Kidney Injury: The ELAIN Randomized Clinical Trial. Jama. 2016;315(20):2190-2199.

43. Gaudry S, Quenot JP, Hertig A, et al. Timing of Renal Replacement Therapy for Severe Acute Kidney Injury in Critically III Patients. American journal of respiratory and critical care medicine. 2019.

44. Narciso RC, Ferraz LR, Mies S, et al. Impact of acute kidney injury exposure period among liver transplantation patients. BMC Nephrology. 2013;14:43-43.

45. Flores A, Asrani SK. The donor risk index: A decade of experience. Liver transplantation: official publication of the American Association for the Study of Liver Diseases and the International Liver Transplantation Society. 2017;23(9):1216-1225.

46. O'Leary JG, Bajaj JS, Tandon P, et al. Outcomes After Listing for Liver Transplant in Patients With Acute-on-Chronic Liver Failure: The Multicenter North American Consortium for the Study of EndStage Liver Disease Experience. Liver transplantation: official publication of the American Association for the Study of Liver Diseases and the International Liver Transplantation Society. 2019;25(4):571-579.

47. EASL Clinical Practice Guidelines for the management of patients with decompensated cirrhosis. $J$ Hepatol. 2018;69(2):406-460.

48. Robb E, Jarman B, Suntharalingam G, Higgens C, Tennant R, Elcock K. Using care bundles to reduce in-hospital mortality: quantitative survey. BMJ. 2010;340:c1234.

\section{Tables}

Table 1 - Basal patient characteristics according to MELD era. Data are expressed as median [IQR] or percentage. BMI: body mass index, APACHE 2: Acute Physiology and Chronic Health Evaluation II, SAPS 3: Simplified Acute Physiology Score III, eGFR: estimated Glomerular filtration rate, MELD: Model for EndStage Liver Disease, CPT: Child-Pugh-Turcote, ICU: Intensive Care Unit 
PRE-MELD ERA POST MELD ERA P value

\begin{tabular}{|c|c|c|c|}
\hline & $N=408$ & $\mathrm{~N}=466$ & \\
\hline Age, years & $52.5[45.25 ; 60]$ & $54[45 ; 61]$ & 0.11 \\
\hline Gender, male (\%) & $266(65)$ & $328(70)$ & 0.18 \\
\hline $\mathrm{BMI}\left(\mathrm{Kg} / \mathrm{m}^{2}\right)$ & $25.2[22.6 ; 28]$ & $25.95[23.1 ; 29.8]$ & 0.02 \\
\hline Hypertension (\%) & $31(8)$ & $139(30)$ & $<0.01$ \\
\hline Diabetes (\%) & $79(8)$ & $139(30)$ & 0.02 \\
\hline APACHE 2 & $16[13 ; 20]$ & $17[15 ; 18]$ & 0.01 \\
\hline SAPS 3 & $39[36 ; 42]$ & $39[28 ; 53]$ & 0.83 \\
\hline Admission eGFR (mL/min/1.73m²) & $91.5[72 ; 110]$ & $88[57 ; 125]$ & 0.46 \\
\hline \multicolumn{4}{|l|}{ Cause of liver disease } \\
\hline Hepatitis C (\%) & $190(47)$ & $209(45)$ & 0.63 \\
\hline Hepatitis B (\%) & $40(10)$ & $30(6)$ & 0.08 \\
\hline Alcoholic liver cirrhosis (\%) & $83(20)$ & $92(20)$ & 0.87 \\
\hline Others (\%) & $75(18)$ & $122(26)$ & 0.01 \\
\hline Hepatocellular carcinoma (\%) & $94(23)$ & $180(39)$ & $<0.01$ \\
\hline Familial amyloid polyneuropathy (\%) & $17(4)$ & $22(5)$ & 0.74 \\
\hline Pre-Transplant Albumin level (g/dL) & $3.1[2.7 ; 3.4]$ & $3[2.6 ; 3.4]$ & 0.14 \\
\hline CPT Score & & & $<0.01$ \\
\hline A or non-cirrhotic (\%) & $70(17)$ & $54(12)$ & \\
\hline $\mathrm{B}(\%)$ & $203(50)$ & $209(45)$ & \\
\hline $\mathrm{C}(\%)$ & $135(33)$ & $203(44)$ & \\
\hline MELD score & $14[10 ; 18]$ & $18[11 ; 25]$ & $<0.01$ \\
\hline Preoperative Creatinine (mg/dL) & $0.9[0.7 ; 1.07]$ & $0.92[0.7 ; 1.32]$ & 0.01 \\
\hline Preoperative Total bilirubin (mg/dL) & $2.6[1.7 ; 4.6]$ & $3[1.8 ; 7 ; 5]$ & $<0.01$ \\
\hline Preoperative prothrombin time, INR & $1.6[1.4 ; 1.96]$ & $1.7[1.35 ; 2.22]$ & 0.14 \\
\hline \multicolumn{4}{|l|}{ Surgical aspects } \\
\hline Deceased donor (\%) & $252(62)$ & $466(100)$ & $<0.01$ \\
\hline Piggyback technique (\%) & $389(95.3)$ & $464(99.6)$ & $<0.01$ \\
\hline
\end{tabular}




\begin{tabular}{llll} 
Operation time (h) & $7.55[6.67 ; 8.5]$ & $6[5.17 ; 6.94]$ & $<0.01$ \\
\hline Total ischemia time (h) & $6.77(2.70 ; 10.15]$ & $9.08[7.87 ; 10.67]$ & $<0.01$ \\
\hline Vasopressor (\%) & $40(9.8)$ & $315(67.6)$ & $<0.01$ \\
\hline Number of blood packs & $4[2 ; 8]$ & $3[2 ; 7]$ & 0.18 \\
\hline Post-transplant care & & & \\
\hline Sepsis during hospitalization (\%) & $157(39)$ & $158(34)$ & 0.18 \\
\hline Vasopressor after transplant (\%) & $70(17)$ & $264(30)$ & $<0.01$ \\
\hline Tacrolimus based immunosuppression $(\%)$ & $299(73)$ & $459(98.5)$ & $<0.01$ \\
\hline First week Tacrolimus peak level (ng/mL) & $12.6[9.9 ; 15.9]$ & $5.7[3.9 ; 7.5]$ & $<0.01$ \\
\hline Acute liver rejection $(\%)$ & $123(30)$ & $69(15)$ & $<0.01$ \\
\hline Nephrotoxic exposure $(\%)$ & $285(70)$ & $152(30)$ & $<0,01$ \\
\hline Urinary output $(\mathrm{ml} / \mathrm{Kg} / \mathrm{H})$ & $0.25[0.15 ; 0.37]$ & $0.55[0.35 ; 0.83]$ & $<0.01$
\end{tabular}

Table 2 - Basal patient characteristics according to AKI occurrence. Data are expressed as median [IQR] or percentage. BMI: body mass index, APACHE 2: Acute Physiology and Chronic Health Evaluation II, SAPS 3: Simplified Acute Physiology Score III, eGFR: estimated glomerular filtration rate 


\begin{tabular}{|c|c|c|c|}
\hline & NON-AKI & AKI & $P$ value \\
\hline & $N=168$ & $N=706$ & \\
\hline Age, years & $55[44 ; 63]$ & $53[45 ; 60]$ & 0.06 \\
\hline Gender, male (\%) & $114(67.9)$ & $480(68)$ & 0.97 \\
\hline $\mathrm{BMI}\left(\mathrm{Kg} / \mathrm{m}^{2}\right)$ & $25.7[22.6 ; 28.5]$ & $25.6[22.9 ; 29.1]$ & 0.38 \\
\hline Hypertension (\%) & $37(22)$ & $133(18.8)$ & 0.35 \\
\hline Diabetes (\%) & $41(24.4)$ & $25.6[22.9 ; 29.1]$ & 0.66 \\
\hline APACHE 2 & $17[14 ; 19]$ & $16[14 ; 19]$ & 0.90 \\
\hline SAPS 3 & $38[30.25 ; 43]$ & $39[32 ; 44,25]$ & $<0.01$ \\
\hline Admission eGFR (mL/min/1.73m²) & $95.5[76.2 ; 118]$ & $90[63 ; 121]$ & 0.01 \\
\hline \multicolumn{4}{|l|}{ Pathogenesis of liver disease } \\
\hline Hepatitis C (\%) & $75(44.6)$ & $324(45.9)$ & 0.77 \\
\hline Hepatitis B (\%) & $17(10.1)$ & $53(7.5)$ & 0.26 \\
\hline Alcoholic liver cirrhosis (\%) & $27(16.1)$ & $148(21)$ & 0.15 \\
\hline Others (\%) & $35(20.8)$ & $162(22.9)$ & 0.56 \\
\hline Hepatocellular carcinoma (\%) & $75(44.6)$ & $199(28.2)$ & $<0.01$ \\
\hline Familial amyloid polyneuropathy (\%) & $11(6.5)$ & $28(4)$ & 0.15 \\
\hline Pre-Transplant Albumin level (g/dL) & $3.1[2.9 ; 3.6]$ & $3[2.7 ; 3.3]$ & $<0.01$ \\
\hline CPT Score & & & $<0.01$ \\
\hline A or non-cirrhotic (\%) & $35(20.8)$ & $89(12.6)$ & \\
\hline $\mathrm{B}(\%)$ & $83(49.4)$ & $329(46.6)$ & \\
\hline $\mathrm{C}(\%)$ & $50(29.8)$ & $288(40.8)$ & \\
\hline MELD score & $13[8 ; 18]$ & $16[11 ; 23]$ & $<0.01$ \\
\hline Preoperative Creatinine (mg/dL) & $0.8[0.7 ; 1.0]$ & $0.9[0.7 ; 1.2]$ & 0.01 \\
\hline Preoperative Total bilirubin (mg/dL) & $2.15[1.4 ; 4.45]$ & $3[1.8 ; 5.9]$ & $<0.01$ \\
\hline Preoperative prothrombin time, INR & $1.5[1.23 ; 1.9]$ & $1.7[1.4 ; 2.15]$ & $<0.01$ \\
\hline \multicolumn{4}{|l|}{ Surgical aspects } \\
\hline Deceased donor (\%) & $150(89.3)$ & $568(80.5)$ & $<0.01$ \\
\hline Piggyback technique (\%) & $161(95.8)$ & $692(98)$ & 0.09 \\
\hline
\end{tabular}




\begin{tabular}{clll} 
Operation time $(\mathrm{h})$ & $6[5.1 ; 7.31]$ & $7[5.67 ; 8]$ & $<0.01$ \\
\hline Total ischemia time $(\mathrm{h})$ & $8.6[6.8 ; 10.6]$ & $8.3[6.4 ; 10.4]$ & 0.19 \\
\hline Vasopressor $(\%)$ & $64(38.1)$ & $291(41.2)$ & 0.49 \\
\hline Number of blood packs & $3[1 ; 9]$ & $4[2 ; 8]$ & 0.31 \\
\hline Post-transplant care & & & \\
\hline Sepsis during hospitalization (\%) & $24(14.3)$ & $291(41.2)$ & $<0.01$ \\
\hline Vasopressor after transplant $(\%)$ & $41(24.4)$ & $293(41.5)$ & $<0.01$ \\
\hline Tacrolimus based immunosuppression $(\%)$ & $153(91.1)$ & $605(85.7)$ & 0.08 \\
\hline First week Tacrolimus peak level $(\mathrm{ng} / \mathrm{mL})$ & $7.7[5.7 ; 11.8]$ & $8.1[4.9 ; 12.5]$ & 0.77 \\
\hline Acute liver rejection $(\%)$ & $18(10.7)$ & $174(24.6)$ & $<0.01$ \\
\hline Nephrotoxic exposure $(\%)$ & $56(33.3)$ & $381(54)$ & $<0.01$ \\
\hline Urinary output $(\mathrm{ml} / \mathrm{Kg} / \mathrm{H})$ & $0.68[0.43 ; 0.97]$ & $0.36[0.21 ; 0.56]$ & $<0.01$
\end{tabular}

Table 3 - Secondary outcomes according to AKI diagnosis. Data are expressed as median [IQR] or percentage. ICU: Intensive care unit, AKI: Acute Kidney Injury, RRT: Renal replacement therapy. 
PRE-MELD ERA POST MELD ERA P value

\begin{tabular}{llll}
\hline & $\mathrm{N}=408$ & $\mathrm{~N}=466$ & \\
\hline Hospital length (days) & $15[11 ; 23]$ & $13[8 ; 22]$ & $<0.01$ \\
\hline ICU length (days) & $3[2 ; 8]$ & $2[1 ; 4]$ & $<0.01$ \\
\hline Retransplant during index hospitalization (\%) & $36(8.8)$ & $36(7.7)$ & 0.62 \\
\hline Acute liver rejection (\%) & $123(30)$ & $69(15)$ & $<0.01$ \\
\hline Acute Kidney Injury (\%) & $342(84)$ & $364(78)$ & 0.04 \\
\hline \multicolumn{1}{c}{ KDIGO 1 } & $171(41.9)$ & $126(27)$ & $<0.01$ \\
\hline \multicolumn{1}{c}{ KDIGO 2 } & $113(27.7)$ & $73(15.7)$ & $<0.01$ \\
\hline Cor 3 & $58(14.2)$ & $165(35.4)$ & $<0.01$ \\
\hline Curation of AKI (days) & $16[10 ; 23]$ & $6[2 ; 23]$ & $<0.01$ \\
\hline Peak creatinine during AKI episode (mg/dL) & $1.6[1.1 ; 2.1]$ & $1.9[1.32 ; 2.69]$ & $<0.01$ \\
\hline Dialysis (\%) & $68(17)$ & $131(28)$ & $<0.01$ \\
\hline \multicolumn{1}{c}{ Intermittent therapies (\%) } & $39(10)$ & $68(15)$ & 0.03 \\
\hline Time of RRT dependence (days) & $50(12)$ & $115(28)$ & $<0.01$ \\
\hline Hospital mortality (\%) & $11.5[3 ; 26.75]$ & $17.5[6 ; 43.75]$ & $<0.01$ \\
\hline 28 days mortality (\%) & $33(8)$ & $36(8)$ & 0.90 \\
\hline 90 days mortality (\%) & $25(6)$ & $45(10)$ & 0.36 \\
\hline 1-year mortality (\%) & $51(13)$ & $45(10)$ & 0.19 \\
\hline & $81(20)$ & $50(11)$ & $<0.01$ \\
\hline
\end{tabular}

Table 4 - Primary and secondary outcomes according to MELD era. Data are expressed as median [IQR] or percentage. ICU: Intensive care unit KDIGO: Kidney disease - Improving Global Outcomes AKI: Acute Kidney Injury RRT: Renal Replacement Therapy 


\begin{tabular}{llll} 
& NON-AKI & AKI & P value \\
\hline & $\mathrm{N}=168$ & $\mathrm{~N}=706$ & \\
\hline Hospital length (days) & $9[7 ; 12]$ & $16[11 ; 25]$ & $<0.01$ \\
\hline ICU length (days) & $2[1 ; 3]$ & $3[2 ; 6]$ & $<0.01$ \\
\hline Retransplant during index hospitalization (\%) & $10(6)$ & $62(8.8)$ & 0.23 \\
\hline Acute liver rejection (\%) & $18(10.7)$ & $174(24.6)$ & $<0.01$ \\
\hline Hospital mortality (\%) & $13(7.7)$ & $56(7.9)$ & 0.93 \\
\hline 28 days mortality (\%) & $14(8.3)$ & $48(6.8)$ & 0.50 \\
\hline 90 days mortality (\%) & $15(8.9)$ & $81(11.5)$ & 0.41 \\
\hline 1-year mortality (\%) & $17(10.1)$ & $114(16.1)$ & 0.05
\end{tabular}

Table 5 - Logistic regression showing unadjusted and 5 distinctive adjusting models for AKI, RRT and 28 days mortality risk between MELD eras. BMI: Body mass index MELD: Model for end-stage liver disease, eGFR: estimated glomerular filtration rate, SAPS 3: Simplified Acute Physiology Score III. 
Predictor

$H R \quad 95 \%$

$\mathrm{Cl}$

$P$ -

value

AKI risk by performing transplantation in Post MELD era

Unadjusted

$0.68 \quad 0.48, \quad 0.03$

0.97

Model 1 - Adjusted for age and gender

$0.69 \quad 0.49, \quad 0.03$

0.97

Model 2 - Adjusted for age, gender, diabetes, hypertension, BMI and MELD

$0.59 \quad 0.41, \quad<0.01$

0.86

Model 3 - Adjusted for age, gender, diabetes, hypertension, BMI, MELD and

$0.60 \quad 0.41, \quad<0.01$ eGFR

Model 4 - Adjusted for age, gender, diabetes, hypertension, BMI, MELD, eGFR,

Vasopressor (During and after transplant), Transfusion, First week

Tacrolimus peak level and Nephrotoxic drug exposure

Model 5 - Adjusted for age, gender, diabetes, hypertension, BMI, MELD, eGFR, Vasopressor (During and after transplant), Transfusion, First week

0.87

$0.46 \quad 0.24, \quad 0.01$

0.85

Tacrolimus peak level, Nephrotoxic drug exposure, Acute liver rejection,

Sepsis and SAPS 3

RRT risk by performing transplantation in Post MELD era

Unadjusted

Model 1 - Adjusted for age and gender

Model 2 - Adjusted for age, gender, diabetes, hypertension, BMI and MELD

Model 3 - Adjusted for age, gender, diabetes, hypertension, BMI, MELD and eGFR

Model 4 - Adjusted for age, gender, diabetes, hypertension, BMI, MELD, eGFR, Vasopressor (During and after transplant), Transfusion, First week

Tacrolimus peak level and Nephrotoxic drug exposure

Model 5 - Adjusted for age, gender, diabetes, hypertension, BMI, MELD, eGFR, Vasopressor (During and after transplant), Transfusion, First week

Tacrolimus peak level, Nephrotoxic drug exposure, Acute liver rejection, Sepsis and SAPS 3

30 days mortality risk by performing transplantation in Post MELD era
$1.95 \quad 1.40, \quad<0.01$

2.71

$1.991 .43,<0.01$

2.78

$1.61 \quad 1.12, \quad<0.01$

2.32

$1.73 \quad 1.20, \quad<0.01$

2.50

1.890 .94

3.80

$2.11 \quad 1.01, \quad 0.04$

4.43

0.07 
Model 2 - Adjusted for age, gender, diabetes, hypertension, BMI and MELD

Model 3 - Adjusted for age, gender, diabetes, hypertension, BMI, MELD and eGFR

0.79 2.44

Model 4 - Adjusted for age, gender, diabetes, hypertension, BMI, MELD, eGFR, Vasopressor (During and after transplant), Transfusion, First week Tacrolimus peak level and Nephrotoxic drug exposure

$0.11 \quad 0.02, \quad<0.01$ 0.52

Model 5 - Adjusted for age, gender, diabetes, hypertension, BMI, MELD, eGFR, Vasopressor (During and after transplant), Transfusion, First week

$0.13 \quad 0.02, \quad<0.01$ Tacrolimus peak level, Nephrotoxic drug exposure, Acute liver rejection, Sepsis and SAPS 3

\section{Figures}




\section{Total: 991}

Excluded (117)

Age $<18$ years $(24)$

Duplicated data (13)

Previous ESRD (23)

Previus Kidney Transplant (18)

Simultaneous Kidney Liver Transplant (19)

Unavailable data (20)

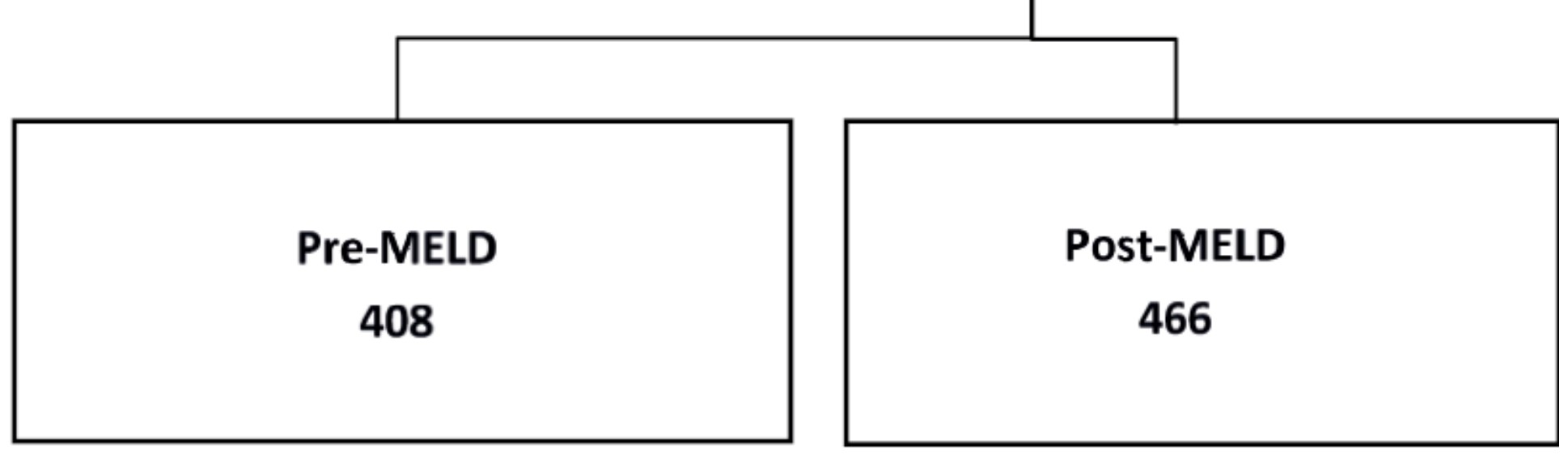

Figure 1

Overview of the study cohort. Flow chart shows patients screened, included and excluded from analysis. ESDR: End-stage renal disease 
AKI risk prediction by MELD era and stratification by KDIGO stages

KDICO 1

$\mathrm{KDIGO} 2$

KDICO 3

Overall
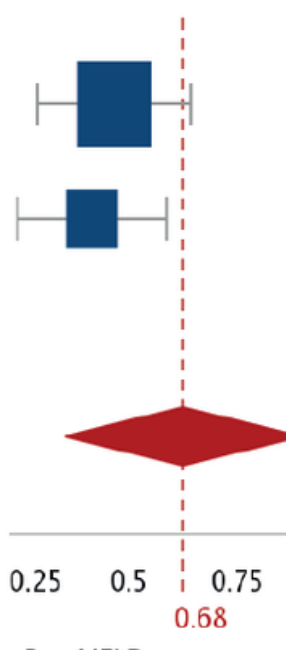

Pre-MELD era
OR $\quad$ LCL $\quad U C L$

$\begin{array}{lll}0.47 & 0.32 & 0.7\end{array}$

$0.41 \quad 0.27 \quad 0.64$

$\begin{array}{lll}1.81 & 1.19 \quad 2.83\end{array}$

$\begin{array}{lll}0.68 & 0.48 \quad 0.97\end{array}$

\section{Figure 2}

AKI risk prediction using 2 periods of me (Pre-MELD and Post MELD) and grouped by KDIGO AKI classification. AKI: Acute Kidney Injury, MELD: Model of End-Stage Liver Disease, KDIGO: Kidney disease improving global outcomes. 

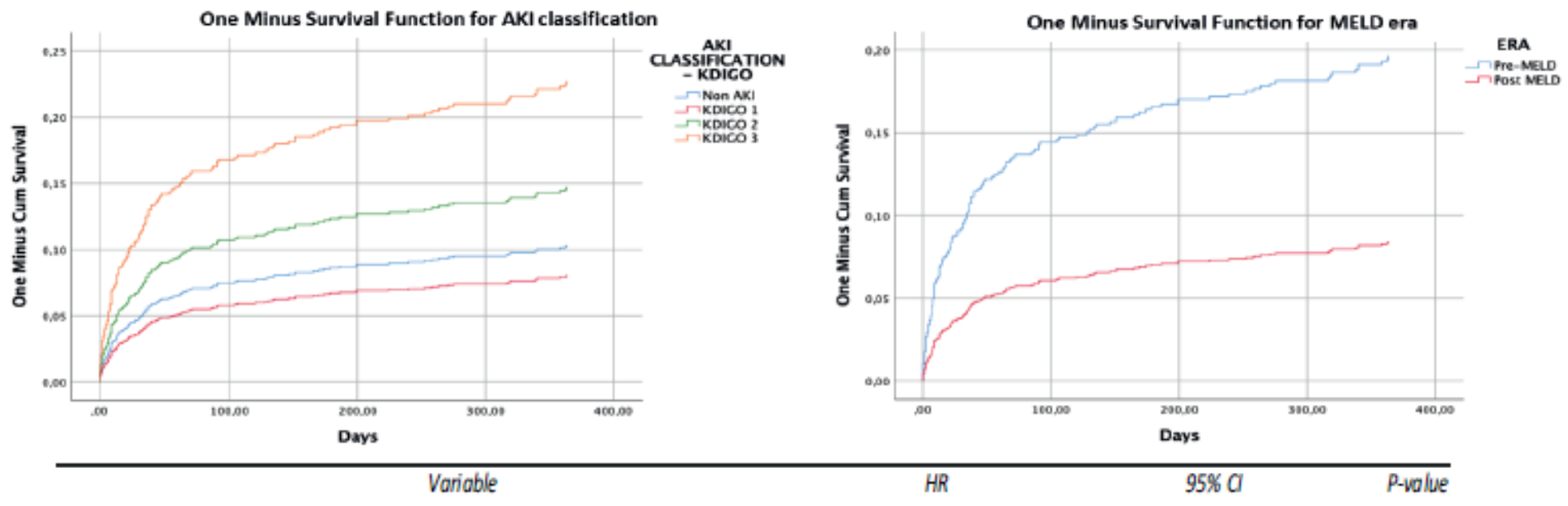

\begin{tabular}{|c|c|c|c|c|}
\hline & Variable & $H R$ & $95 \% a$ & P-value \\
\hline \multicolumn{5}{|c|}{365 motality risk - Cox proporfonal hazards analysis } \\
\hline \multicolumn{2}{|c|}{ Gender (male as reference) } & 0.74 & $0.52,1.07$ & 0.11 \\
\hline \multicolumn{2}{|c|}{ Age (1 year) } & 0.99 & $0.98,1.01$ & 0.84 \\
\hline \multicolumn{2}{|l|}{ SAPS3 (1 point) } & 1.00 & $0.98,1.01$ & 0.76 \\
\hline \multicolumn{2}{|l|}{ Hypettension } & 0.64 & $0.36,1.14$ & 0.13 \\
\hline \multicolumn{2}{|l|}{ Diabetes melitis } & 121 & $0.78,1.86$ & 0.38 \\
\hline \multicolumn{2}{|l|}{ BMI $\left(1 \mathrm{Kg} / \mathrm{m}^{2}\right)$} & 0.99 & $0.95,1.03$ & 0.79 \\
\hline \multicolumn{2}{|c|}{ MELDERA Pre-NELD as reference) } & 0.40 & $0.26,0.60$ & $<0.01$ \\
\hline \multicolumn{2}{|c|}{ MELD (1 point) } & 1.02 & $1.01,1.04$ & $<0.01$ \\
\hline \multirow[t]{3}{*}{ KDIGO dassifcafion } & Non-AKI & ref & & \\
\hline & $\begin{array}{l}\text { KDIGO } 1 \\
\text { KDIGO } 2\end{array}$ & $\begin{array}{l}0.76 \\
1.45\end{array}$ & $\begin{array}{l}0.41,1.41 \\
0.84,264\end{array}$ & $\begin{array}{l}0.36 \\
0.21\end{array}$ \\
\hline & KDIGO3 & 236 & $1.33,4.18$ & $<0.01$ \\
\hline \multicolumn{2}{|c|}{ Hepatoceluluar carcinoma } & 1.53 & $1.02,2.30$ & 0.03 \\
\hline \multicolumn{2}{|c|}{ Acute liver rejection } & 1.02 & $0.69,1.52$ & 0.89 \\
\hline
\end{tabular}

Figure 3

Cox proportional hazard analysis for 1-year mortality. SAPS3: Simplified Acute Physiology Score III, BMI: Body mass index, KDIGO: Kidney disease - Improving Global Outcomes, AKI: Acute Kidney Injury 